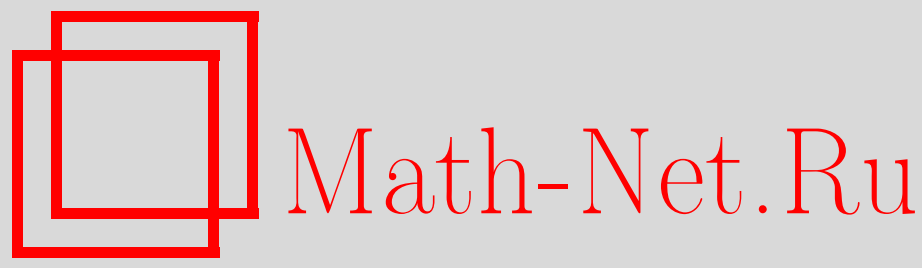

А. В. Анашкин, Об одном обобщении теоремы Орэ для многочленов, Дискрет. матем., 2015, том 27, выпуск $4,21-25$

DOI: https://doi.org/10.4213/dm1344

Использование Общероссийского математического портала Math-Net.Ru подразумевает, что вы прочитали и согласны с пользовательским соглашением http://www.mathnet.ru/rus/agreement

Параметры загрузки:

IP : 3.82 .47 .9

26 апреля 2023 г., $16: 29: 32$

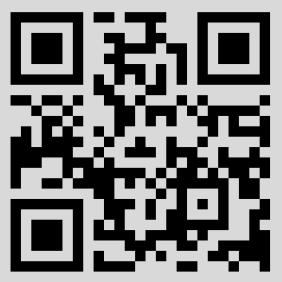




\title{
Об одном обобщении теоремы Орэ для многочленов
}

\author{
() 2015 г. А. В. Анашкин*
}

Пусть $G F(q)$ - поле из $q$ элементов, а $V_{n}(q)$ - множество всех $n$-мерных векторов над полем $G F(q)$. Линеаризованным многочленом, соответствующим многочлену $f(x)=x^{n}-\sum_{i=0}^{n-1} c_{i} x^{i}$ над полем $G F(q)$, называется многочлен $F(x)=$ $x^{q^{n}}-\sum_{i=0}^{n-1} c_{i} x^{q^{i}}$. Рассматривается преобразование $T_{f}$ пространства векторов $V_{n}(q)$, действующее по правилу $T_{f}\left(\left(u_{0}, \ldots, u_{n-2}, u_{n-1}\right)\right)=\left(u_{1}, \ldots, u_{n-1}, \sum_{i=0}^{n-1} c_{i} u_{i}\right)$. Доказывается, что если $c_{0} \neq 0$, то граф преобразования $T_{f}$ изоморфен графу преобразования $Q: \alpha \rightarrow \alpha^{q}$ на множестве всех корней многочлена $F(x)$ в поле его разложения. При этом граф преобразования $T_{f}$ состоит из циклов длин $1 \leqslant d_{1} \leqslant d_{2} \leqslant \ldots \leqslant d_{r}$ тогда и только тогда, когда многочлен $F(x)$ является произведением $r+1$ неприводимых многочленов, которые имеют степени $1, d_{1}, d_{2}, \ldots, d_{r}$.

Ключевые слова: линеаризованный многочлен, примитивный многочлен, изоморфизм графов, теорема Орэ

Будем использовать следующие обозначения: $P=G F(q)$ - поле из $q$ элементов, $q$ - степень простого числа; $P[x]$ - кольцо многочленов над полем $P ; V_{n}(q)$ - векторное пространство размерности $n$ над полем $P$, элементы которого - векторы размерности $n$ над $P, \tau: P[x] \rightarrow P[x]$ - отображение, задаваемое формулой $\tau\left(\sum_{i=0}^{n} c_{i} x^{i}\right)=\sum_{i=0}^{n} c_{i} x^{q^{i}}$

По многочлену $f(x)=x^{n}-\sum_{i=0}^{n-1} c_{i} x^{i}$ над полем $P$ определим линейное отображение $T_{f}: V_{n}(q) \rightarrow V_{n}(q)$, действующее по правилу $T_{f}\left(\left(u_{0}, \ldots, u_{n-2}, u_{n-1}\right)\right)=$ $\left(u_{1}, \ldots, u_{n-1}, \sum_{i=0}^{n-1} c_{i} u_{i}\right)$. Известно, что в случае $c_{0} \neq 0$ граф преобразования $T_{f}$ представляет собой объединение нескольких циклов и, в частности, указанный граф содержит как минимум один цикл единичной длины, поскольку $T_{f}((0,0, \ldots, 0))=$ $(0,0, \ldots, 0)$. Если граф преобразования $T_{f}$ состоит всего из двух циклов, имеющих длины 1 и $q^{n}-1$, то многочлен $f(x)$ называют примитивным.

*Место работы: Лаборатория ТВП, e-mail: alexander.anashkin@bk.ru 
Критерий примитивности многочлена $f(x)$ дает теорема Орэ-Глисона-Марша [2],[4], являющаяся следствием более общей теоремы Орэ [4]. Теорема Орэ гласит, что если многочлен $f(x)$ является неприводимым, то степень любого неприводимого делителя многочлена $\frac{\tau(f(x))}{x}$ равна периоду многочлена $f(x): \operatorname{ord}(f(x))=$ $\min \left\{t \in \mathbb{N} \mid f(x)\right.$ делит $\left.\left(x^{t}-1\right)\right\}$. В условиях теоремы Орэ граф преобразования $T_{f}$ состоит из одного цикла длины 1 и $\frac{q^{n}-1}{\operatorname{ord}(f(x))}$ циклов длины $\operatorname{ord}(f(x))$. Теорема Орэ-Глисона-Марша соответствует случаю ord $(f(x))=q^{n}-1$.

Теорему Орэ-Глисона-Марша можно рассматривать и как критерий неприводимости многочлена $F(x)=x^{q^{m}-1}+a_{m-1} x^{q^{m-1}-1}+\ldots+a_{1} x^{q-1}+a_{0}, a_{0} \neq 0$, над полем $P=G F(q)$. В [3] содержится обобщение этой теоремы - критерий неприводимости многочлена $F(x)=x^{q^{m}-1}+a_{m-1} x^{q^{m-1}-1}+\ldots+a_{1} x^{q-1}+a_{0}, a_{0} \neq 0$, над полем $G F\left(q^{t}\right)$ в случае $t>1$.

Далее мы обобщим теорему Орэ на случай не обязательно неприводимого многочлена $f(x)$ над полем $P, f(0) \neq 0$. Указанное обобщение будет получено в виде следствия из построения изоморфизма двух векторных пространств.

Пусть, как и ранее, $f(x)=x^{n}-\sum_{i=0}^{n-1} c_{i} x^{i} \in P[x], c_{0} \neq 0, \widehat{P}-$ поле разложения многочлена $F(x)=\tau(f(x))$ над полем $P$, а $M_{f}$ - множество всех корней многочлена $F(x)$ в поле $\widehat{P}: M_{f}=\{\alpha \in \widehat{P} \mid F(\alpha)=0\}$.

Известно, что $M_{f}$ является векторным пространством над полем $P$ размерности $n$, а отображение $Q: \widehat{P} \rightarrow \widehat{P}$, заданное по правилу $Q(\alpha)=\alpha^{q}$, является биективным и линейным, более того, $Q\left(M_{f}\right)=M_{f}[1]$.

Лемма 1. B пространстве $M_{f}$ существует такой элемент $\beta$, что система элементов $\beta_{0}=\beta, \beta_{1}=Q(\beta), \beta_{2}=Q^{2}(\beta), \ldots, \beta_{n-1}=Q^{n-1}(\beta)$ является базисом.

Доказательство леммы 1 следует из теорем 3.65 и 3.72 [1].

Лемма 2. Если $c_{0} \neq 0$, то в векторном пространстве $V_{n}(q)$ существует такой базис $\alpha_{0}, \ldots, \alpha_{n-1} \in V_{n}(q)$, что $a_{i+1}=T_{f}\left(\alpha_{i}\right), i=0, \ldots, n-2$, и для вектора $\alpha_{n}=$ $T_{f}\left(\alpha_{n-1}\right)$ выполняется равенство $\alpha_{n}=\sum_{i=0}^{n-1} c_{i} \alpha_{i}$.

Доказательство леммы 2. Легко видеть, что система векторов $\alpha_{0}, \ldots, \alpha_{n-1}$, где $\alpha_{0}=(0,0, \ldots, 0,1)$ и $\alpha_{i}=\left(T_{f}\right)^{i}\left(\alpha_{0}\right), i=1, \ldots, n-1$, является базисом, а из определения отображения $T_{i}$ и вектора $\alpha_{n}$ получаем, что $\alpha_{n}=T_{f}\left(\alpha_{n-1}\right)=\sum_{i=0}^{n-1} c_{i} \alpha_{i}$.

Приведенные в леммах 1 и 2 базисы называются циклическими относительно соответствующих преобразований.

Теорема 1. Пусть $f(x)=x^{n}-\sum_{i=0}^{n-1} c_{i} x^{i} \in P[x], c_{0} \neq 0, \widehat{P}-$ поле разложения многочлена $F(x)=\tau(f(x))$ над полем $P, M_{f}$ - множество всех корней многочлена $F(x)$ в поле $\widehat{P}: M_{f}=\{\alpha \in \widehat{P} \mid F(\alpha)=0\}$, отображение $Q: M_{f} \rightarrow M_{f}$ задается по правилу $Q(\alpha)=\alpha^{q}$. Тогда существует такой изоморфизм $\varphi: V_{n}(q) \rightarrow M_{f}$ векторных пространств, что для любого вектора $a \in V_{n}(q)$ справедливо равенство $Q(\varphi(a))=\varphi\left(T_{f}(a)\right)$, т.е. коммутативна диаграмма 


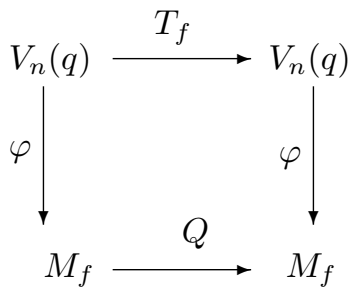

Доказательство теоремы 1. Согласно леммам 1 и 2 существуют циклический базис $\alpha_{0}, \alpha_{1}, \ldots, \alpha_{n-1}$ пространства $V_{n}(q)$ относительно преобразования $T_{f}$ и циклический базис $\beta_{0}, \beta_{1}, \ldots, \beta_{n-1}$ пространства $M_{f}$ относительно преобразования $Q$.

Рассмотрим линейное отображение $\varphi: V_{n}(q) \rightarrow M_{f}$, определенное равенствами $\varphi\left(\alpha_{i}\right)=\beta_{i}, i=0, \ldots, n-1$. Такое отображение существует и единственно. $\mathrm{B}$ силу определения $\varphi$ достаточно доказать утверждение теоремы только для базисных векторов.

Для $i=0, \ldots, n-2$ по заданию отображения $\varphi$ имеем равенства $Q\left(\varphi\left(\alpha_{i}\right)\right)=$ $Q\left(\beta_{i}\right)=\beta_{i+1}=\varphi\left(\alpha_{i+1}\right)=\varphi\left(T_{f}\left(\alpha_{i}\right)\right)$.

Далее, обозначив $\beta=\beta_{0}$, находим, что $Q\left(\varphi\left(\alpha_{n-1}\right)\right)=Q\left(\beta_{n-1}\right)=Q\left(\beta^{q^{n-1}}\right)=\beta^{q^{n}}=$ $\sum_{i=0}^{n-1} c_{i} \beta^{q^{i}}=\sum_{i=0}^{n-1} c_{i} \beta_{i}$, так как $F(\beta)=0$ и $F(x)=x^{q^{n}}-\sum_{i=0}^{n-1} c_{i} x^{q^{i}}$. По лемме 2 имеем равенство $\varphi\left(T_{f}\left(a_{n-1}\right)\right)=\varphi\left(\sum_{i=0}^{n-1} c_{i} \alpha_{i}\right)=\sum_{i=0}^{n-1} c_{i} \beta_{i}$, которое завершает доказательство теоремы.

Следствие 1. В условиях теоремы 1 число ииклов длины d в графе линейного преобразования $T_{f}$ равно числу неприводимых сомножителей степени $d$ в разложении многочлена $F(x)=\tau(f(x))$.

Доказательство следствия 1 вытекает из теоремы 1 и двух фактов.

(1) Для любого многочлена $g(x) \in P[x]$ если $\alpha$ - корень многочлена в поле разложения, то и $\alpha^{q}$ - корень многочлена $g(x)$.

(2) Степень неприводимого многочлена $g(x) \in P[x]$ равна минимальному периоду последовательности $\alpha, \alpha^{q}, \alpha^{q^{2}}, \alpha^{q^{3}}, \ldots$, где $\alpha$ - корень многочлена $g(x)$.

Следствие 2 (Теорема Орэ-Глисона-Марша [2; стр. 236], [4; стр. 77]). Пусть $f(x)=x^{n}-\sum_{i=0}^{n-1} c_{i} x^{i} \in P[x], c_{0} \neq 0, F(x)=\tau(f(x))=\widetilde{F}(x) \cdot x$. Многочлен $f(x)$ - примитивный тогда и толъко тогда, когда $\widetilde{F}(x)$ - неприводимый.

Теорема 1 допускает обобщение на случай произвольного значения $c_{0}$. Пусть $t$ - максимальное целое неотрицательное число для которого многочлен $x^{t}$ делит многочлен $f(x)$. В [1] показано, что если $F(x)=\tau(f(x))=$ $\prod_{i=0}^{q^{n}-1}\left(x-\xi_{i}\right)$ - разложение многочлена $F(x)$ на линейные множители в поле разложения $\widehat{P}, \xi_{i} \in \widehat{P}$, то каждый корень встречается в разложении многочлена $F(x)$ ровно $q^{t}$ раз: $F(x)=\left(\prod_{i=0}^{q^{n-t}-1}\left(x-\xi_{i}^{*}\right)\right)^{q^{t}} \cdot$ Пусть $M_{f}^{*}=$ $\left\{\xi_{i}^{*} \in \widehat{P}, i=0, \ldots, q^{n-t}-1 \mid F\left(\xi_{i}^{*}\right)=0, \xi_{i}^{*} \neq \xi_{j}^{*}, i \neq j\right\}-$ множество всех различных 
корней многочлена $F(x)$. Тогда множество $M_{f}$ всех корней многочлена $F(x)$ с учетом их кратности можно рассматривать как декартово произведение двух множеств: $M_{f}=\left\{(\alpha, s) \mid \alpha \in M_{f}^{*}, s \in\left\{1,2, \ldots, q^{t}\right\}\right\}=M_{f}^{*} \times\left\{1,2, \ldots, q^{t}\right\}$.

Теорема 2. Существуют такое отображение $Q^{*}: M_{f} \rightarrow M_{f}$ удовлетворяющее условию: если $Q^{*}((\alpha, x))=(\beta, y), \alpha, \beta \in M_{f}^{*}, x, y \in 1, \ldots, q^{t}$, mо $\beta=\alpha^{q}$, и такое биективное отображение $\varphi: V_{n}(q) \rightarrow M_{f}$, что для любого вектора $a \in V_{n}(q)$ справедливо равенство $Q^{*}(\varphi(a))=\varphi\left(T_{f}(a)\right)$, т.е. коммутативна диаграмма

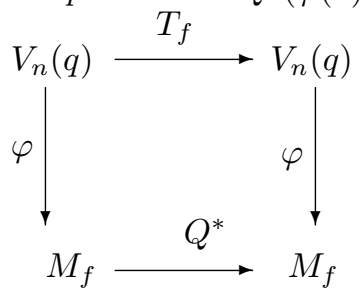

Доказательство теоремы 2 производится по аналогии с доказательством теоремы 1 и с её использованием. Оно не представляет сложности и для краткости изложения мы его не приводим.

Пример 1. $q=2, f(x)=\left(x^{2}+x+1\right) \cdot x=x^{3}+x^{2}+x$.

$F(x)=\tau(f(x))=x^{8}+x^{4}+x^{2}=\left(\left(x^{3}+x+1\right) \cdot x\right)^{2}$. Пусть $M_{f}^{*}=\{\alpha, \beta, \gamma, 0\}, \alpha, \beta, \gamma$ - корни многочлена $x^{3}+x+1$ в поле разложения. Тогда граф преобразования $\delta \mapsto \delta^{q}$ на множестве $M_{f}^{*}$ имеет вид
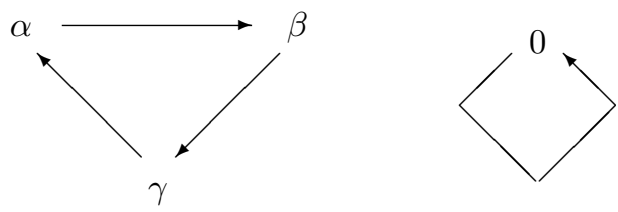

При этом

$$
M_{f}=\{(\alpha, 0),(\beta, 0),(\gamma, 0),(0,0),(\alpha, 1),(\beta, 1),(\gamma, 1),(0,1)\} .
$$

Граф отображения $Q^{*}$ в этом случае имеет вид
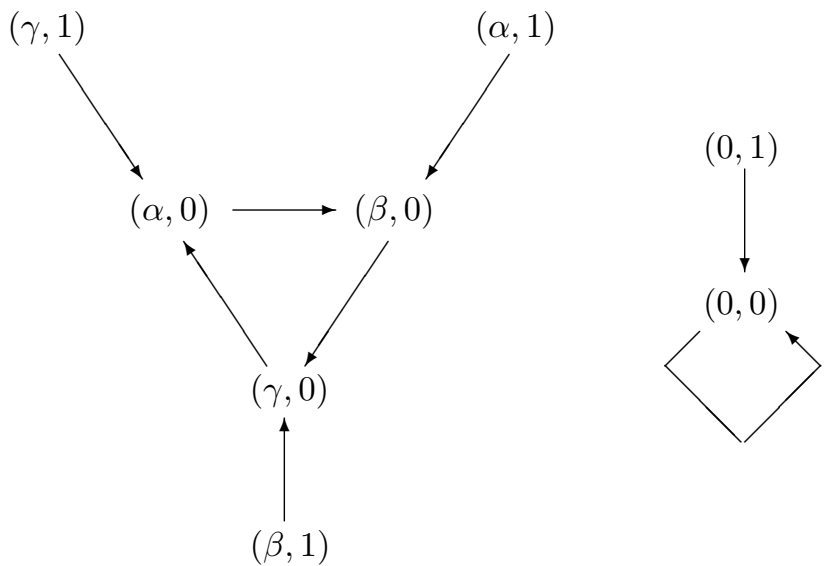
В точности такую же структуру имеет и граф преобразования $T_{f}: V_{3} \rightarrow V_{3}$ : $T_{f}\left(\left(a_{0}, a_{1}, a_{2}\right)\right)=\left(a_{1}, a_{2}, a_{1}+a_{2}\right)$.

Автор выражает искреннюю благодарность А.А. Нечаеву за полезные замечания, сделанные в ходе обсуждения работы, и посвящает статью светлой памяти безвременно ушедшего учителя.

\section{Список литературы}

1. Лидл Р., Нидеррайтер Г., Конечные поля, М.: «Мир», 1988.

2. Глухов М.М., Елизаров В.П., Нечаев А.А., Алгебра, М.: «Гелиос АРВ», 2003.

3. Нечаев А.А., Попов В.О., "Обобщение теоремы Орэ о неприводимых многочленах над конечным полем", Дискретная математика, 27:1 (2015), 108-110.

4. Цирлер Н., "Линейные возвратные последовательности", Кибернетический сборник, 1963, № 6, 55-79. 